\title{
GPS phase fluctuations observed along the American sector during low irregularity activity months of 1997-2000
}

\author{
S. J. Shan ${ }^{1}$, J. Y. Liu ${ }^{1}$, F. S. Kuo ${ }^{1}$, C. C. Liu ${ }^{2}$, and H. F. Tsai ${ }^{1}$ \\ ${ }^{1}$ Institute of Space Sciences, National Central University, Chung-Li, Taiwan \\ ${ }^{2}$ Institute of Earth Sciences, Academia Sinica, Taipei, Taiwan
}

(Received May 30, 2001; Revised November 14, 2001; Accepted November 16, 2001)

\begin{abstract}
The Global Positioning System (GPS) provides an alternative way to investigate ionospheric irregularities and their effects on the radio wave propagation. The method is based on fluctuations of the total electron content (TEC) resulted from the ionospheric plasma irregularities. Previous studies have showed the correlation between the radiowave intensity (including GPS signals) and ionospheric irregularities during magnetic storm periods. In this study, phase fluctuations derived from GPS signals are used to address aspects of the ionospheric storm events during the low irregularity activity months. We analyze data from seven GPS stations located in Central- and SouthAmerica during eight magnetic storms occurred from 1997 to 2000. It is found that, in general no significant feature in the phase fluctuation is observed during the low irregularity activity months, except during the 26 August 1998 and the 15 July 2000 storms. A detailed study shows that the GPS phase fluctuations develop when the Dst index begins to decrease significantly. This phenomenon cannot be compared directly to previous observations and model results due to the fundamental difference in the background levels of irregularity activity. To better understand the generation of ionospheric irregularities during the storm period of the low irregularity activity months, the temporal relationship between the magnetic Dst index, equatorial anomaly TEC, and the GPS phase fluctuations are examined and discussed.
\end{abstract}

\section{Introduction}

In the past two decades, numerous studies, including radar, satellite, and rocket observations, have proven that ionospheric irregularities often exist in the equatorial regions (cf., Kelley, 1989; Pi et al., 1997). More recent studies suggested that the development of ionospheric irregularities might correlate with the occurrence of magnetic storm (Aarons et al., 1997; Pi et al., 1997). Although many previous studies tried to model the relationship between ionospheric irregularities and magnetic storms (Mullen, 1973; Basu et al., 1988; Dabas et al., 1988; Aarons, 1991), the detailed configuration still remains unclear.

Scintillation techniques have been developed by observing fluctuations in the amplitude and phase of satellite beacon signals propagating through the ionospheric irregularities (Aarons, 1993). The establishment of the Global Positioning System (GPS) provides an alternative way to investigate the ionospheric irregularities. Ho et al. (1996) examined the total electron content (TEC) from more than 60 worldwide GPS stations. They found significant increases in TEC along Greenwich meridian in the northern hemisphere during the magnetic storm on 26 November 1994, while Jakowski et al. (1999) and Liu et al. (1999) observed a large TEC deviation from the associated average during the magnetic storm on 10 January 1997. Aarons et al. (1997), using the GPS phase fluctuation (which is actually the time

Copy right (c) The Society of Geomagnetism and Earth, Planetary and Space Sciences (SGEPSS); The Seismological Society of Japan; The Volcanological Society of Japan; The Geodetic Society of Japan; The Japanese Society for Planetary Sciences. variation of TEC) as an indicator of the occurrence of ionospheric irregularities, studied several magnetic storms from 1993 to 1995, and found that the strength and occurrence of irregularities increased during the storm periods. They concluded that apparent GPS phase fluctuations could be observed when the intensities of magnetic storms reach their maxima (as indicated by the Dst minima).

Based on satellite in-situ data, scintillation, and spreadF observations, Aarons (1993) suggested that, in addition to the latitude, the development of ionospheric irregularities shows also a clear seasonal trend depending on the longitude. For example, the occurrence frequency of ionospheric irregularities in South America is significantly lower during the winter season (i.e., May-August) than during the summer, whereas the occurrence frequency of ionospheric irregularities in Central Pacific has the exactly opposite pattern (Aarons, 1993; Maruyama and Matuura, 1984; Maruyama, 1988; Wanninger, 1993). These studies suggested that the phase fluctuations observed during those specific magnetic storms also occurred during the seasons favoring the development of ionospheric irregularities. In other words, both seasonal trend and existence of magnetic storms are positive factors in producing the irregularities.

In this paper, we investigate the phase fluctuations appearing during the magnetic storm period in the season of low ionospheric irregularity activity. Thus, this allows us to identify whether the seasonal trend or the occurrence of magnetic storms is more predominant in producing ionospheric irregularities. To answer the questions above, TECs 


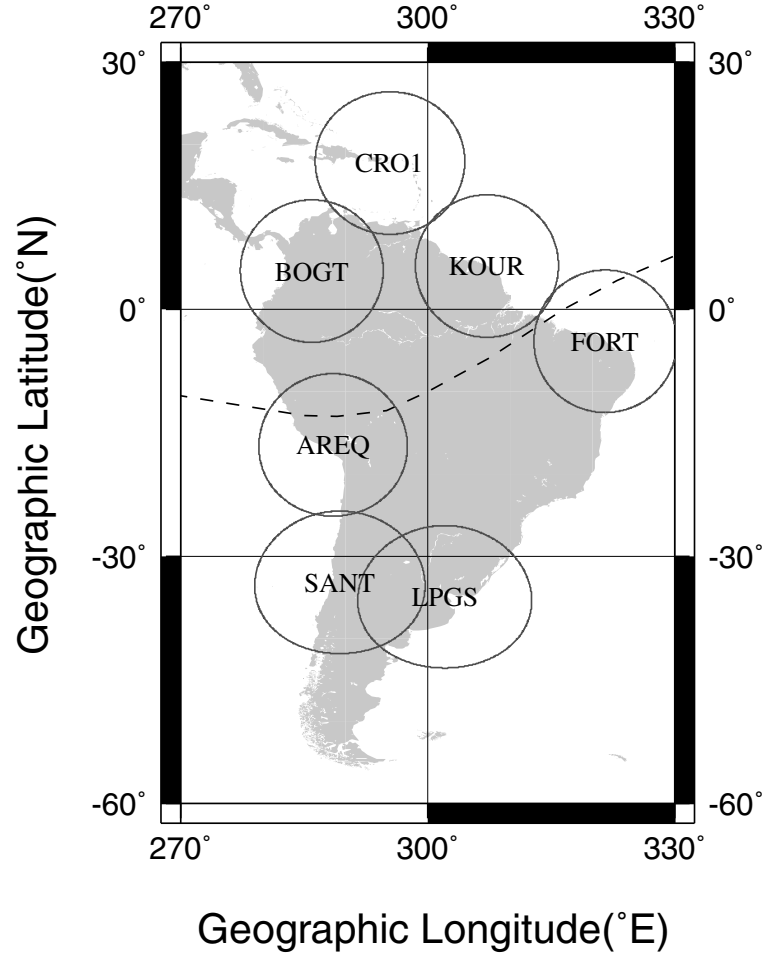

Fig. 1. The locations and coverage of seven ground-based receivers of the GPS network. The circle at each site gives the field of view for a $350 \mathrm{~km}$ intersection height at an elevation angle at $20^{\circ}$. Dashed lines represent the magnetic equator.

derived from a network of seven ground-based GPS stations in Central-South America from 1997 to 2000 are examined (Fig. 1). During the period, eight magnetic storms, with their Dst smaller than $-100 \mathrm{nT}$, occurred in the low irregularity activity months (May to August). Therefore, these eight magnetic storms provide an opportunity to identify the controlling factors. Finally, based on the relationship between the variations of magnetic storms and phase fluctuations, we propose that generating irregularities in a low occurrence season requires two conditions: (1) the magnetic Dst index must begin to sharply decrease during pre- and post-sunset periods, and (2) the existence of relatively intense equatorial ionization anomaly.

\section{Observation and Analysis}

The GPS is ideally suited for a simultaneous monitoring a large area of the ionosphere. The system consists of more than 24 satellites, evenly distributed in 6 orbital planes around the globe at an altitude of about 20,200 km. Each satellite transmits two frequencies of signals $\left(f_{1}=1575.42\right.$ $\mathrm{MHz}$, and $f_{2}=1227.60 \mathrm{MHz}$ ). Since the ionosphere is a dispersive medium, scientists are able to evaluate the ionospheric TEC by measuring the modulations on carrier phases and phase codes recorded by dual-frequency receivers (Leick, 1995; Sardón et al., 1994; Liu et al., 1996; Zarraoa and Sardón 1996). Here we retrieve data from the International GPS Service (IGS), apply the technique of Liu et al. (1996), and convert the slant TEC into the vertical TEC by given recorded broadcast ephemeris and a suitable subionospheric height (cf., Tsai and Liu, 1999).
Aarons et al. (1996, 1997) and Pi et al. (1997) analyzed the rate of change of GPS TEC, i.e., dTEC/dt, at 1-min intervals, to get an insight into the ionospheric irregularities. Although the 30 seconds sampling rate of the GPS data has a limited spectral bandwidth to detect the amplitude scintillations originating from equatorial irregularities, Beach and Kintner (1999) demonstrated its ability to identify the irregularities through GPS phase fluctuations. In this study, we adopt the method employed by several research groups (Aarons et al., 1996, 1997; Pi et al., 1997), and apply a Butterworth high-pass filter with a corner frequency of 0.00067 $\mathrm{Hz}$ (i.e., 25 minutes) to each trace of one-day length to remove the long-period variation.

To quantitatively characterize the phase fluctuations, we adopt the scheme outlined by Mendillo et al. (2000) and calculate the indices $f p$ and $F p$ from the high-passed traces. The index $f p$ is defined as the median of the 60-sec phase fluctuation data (in absolute value) from one satellite over a 15 -min period. These $f p$ values are then averaged over a period of one hour for all satellites available to one station, after multiplied by 1000 , to give the integer index $F p$, which represents the average level of irregularities in the hour above that specific station. In general, the background noise level of irregularities has an $F p$ value of $<50$. The $F p$ value between 50 and 200 represents the existence of moderate irregularities, while $F p>200$ indicates the occurrence of very strong irregularity levels (Mendillo et al., 2000).

\section{Results}

Figure 1 shows the locations of ground-based GPS stations and the associated encompassed coverage. The coordinates of these stations are listed in Table 1. Figure 2 displays the monthly averaged $F p$ from the seven GPS ground stations in 1998. It is clear that the monthly averaged $F p$ are low during May-August 1998. Figures 3, 4, and 5 show the hourly $F p$ values and the associated $K p$ and $D s t$ indices of the eight magnetic storms occurring during the low irregularity activity months of 1997-2000. Table 2 summarizes time variations of the magnetic index Dst and the number of proportion of the GPS stations detecting the phase fluctuations at certain $F p$ levels for each storm event. Ts, Tb, and Tm denote the SSC onset times, the Dst beginning to sharply decrease, and the Dst reaching its minimum, respectively, while Tp represents the starting time of the phase fluctuations. Note that the GPS measurements have some data gaps and therefore the number of the stations available for each storm event are different. For example, the number of proportion $1 / 7 \mathrm{M}(1 / 7 \mathrm{~S})$ denotes one out of seven stations detecting Moderate fluctuations $100 \leq F p \leq 200$ (the Strong fluctuations, $F p>200$ ). Note that in this study, to ensure the occurrence of phase fluctuations we require $F p \geq 100$ to be significant, which differs slightly from $F p \geq 50$ suggested by Mendillo et al. (2000). The universal time (UT) coordinate is adopted by the GPS, geomagnetic storm table, and magnetic index, while the local time (LT) coordinate is useful for geophysical studies. Note that due to the scattered locations, UT leads LT of these stations by varying from 2 to 5 hours (LT $=$ UT + (station's geographic longitude in ${ }^{\circ} \mathrm{E} / 15^{\circ}$ ) $\mathrm{hr}$; see Table 1 for details). In this study, both time coordinates are employed. 
Table 1. Locations of the GPS stations.

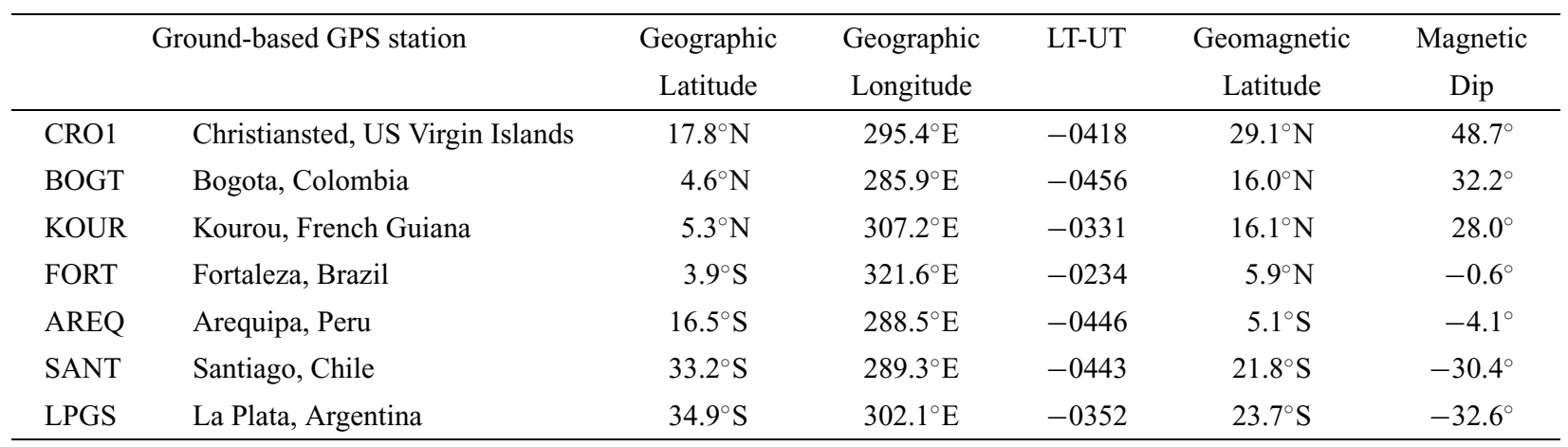
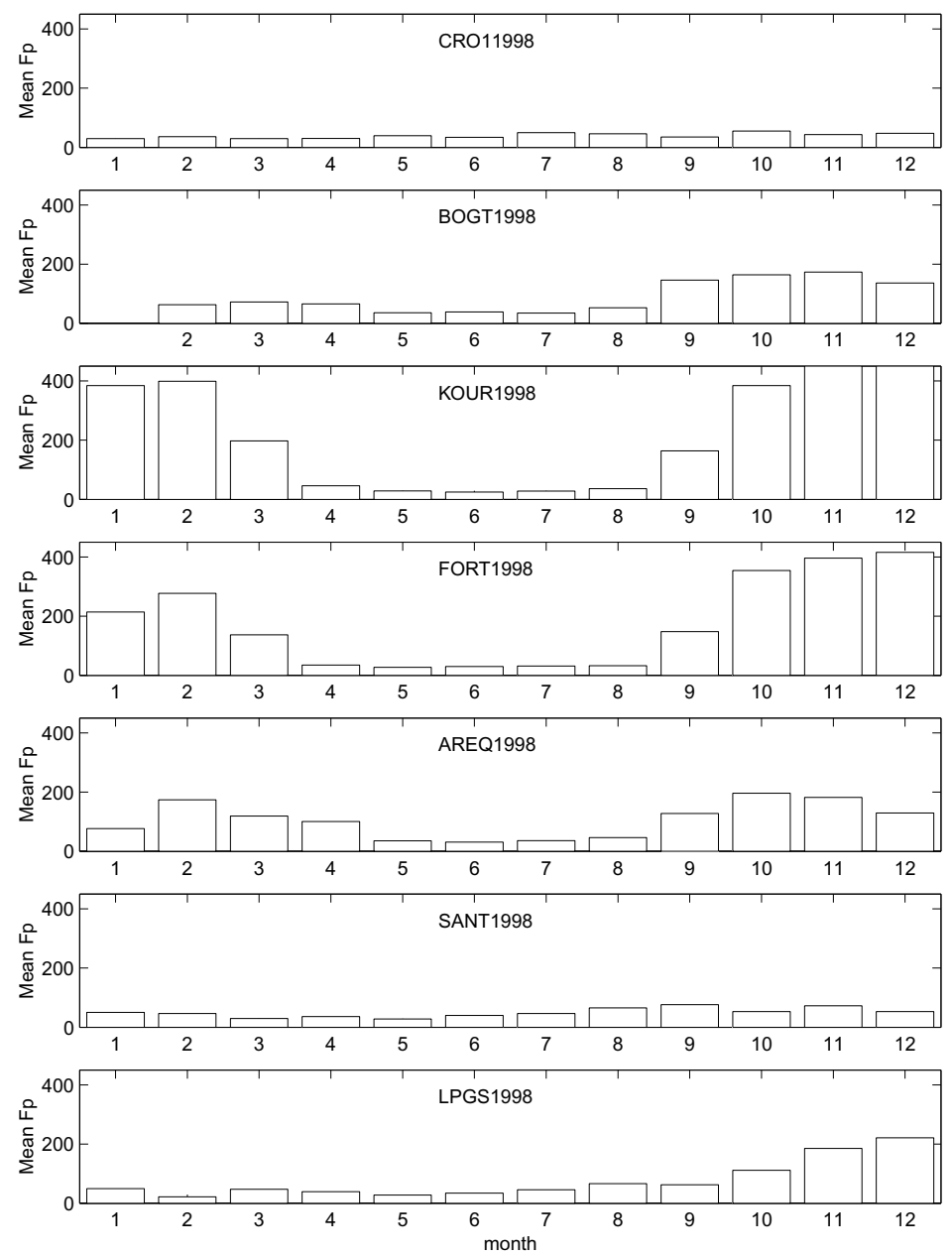

Fig. 2. The monthly averaged $F p$ from the seven GPS ground stations in 1998. The values in November and December, 1998, for station KOUR are both off-scaled (714 and 661, respectively).

Figures 3, 4, and 5 show that some background levels of irregularities $F p<50$ indicated by Mendillo et al. (2000) appear between 1200 and 2400 UT. It can be seen that no clear phase fluctuations with $F p>100$ appear in Events A, $\mathrm{B}$, and $\mathrm{C}$; one station observes the phase fluctuations during Events D (station BOGT), E (station KOUR), and F (station KOUR); and the most pronounced features occur during Events $\mathrm{G}$ and $\mathrm{H}$, respectively (also see Table 2). Figure 4(a) illustrates that the moderate phase fluctuations at BOGT increase at 0200 UT (2100 LT) while the Dst index decreases at about 0000 UT (1900 LT, post-sunset) on 26 June 1998, respectively. Figure 4(b) shows that the moderate phase fluctuations with a short duration at KOUR start to increase at 0500 UT $(0130 \mathrm{LT})$ when the Dst decreases at about 0300 UT (about 2330 LT around midnight) on 24 May 2000. There are two Dst decreases in Event F. It is interesting to notice that the moderate phase fluctuations occur at KOUR at 2300 UT associated with the first (smaller) decrease at 2100 UT (1730 LT, pre-sunset) on 10 August but no clear fluctuation is detected during the second (greater) $D s t$ 

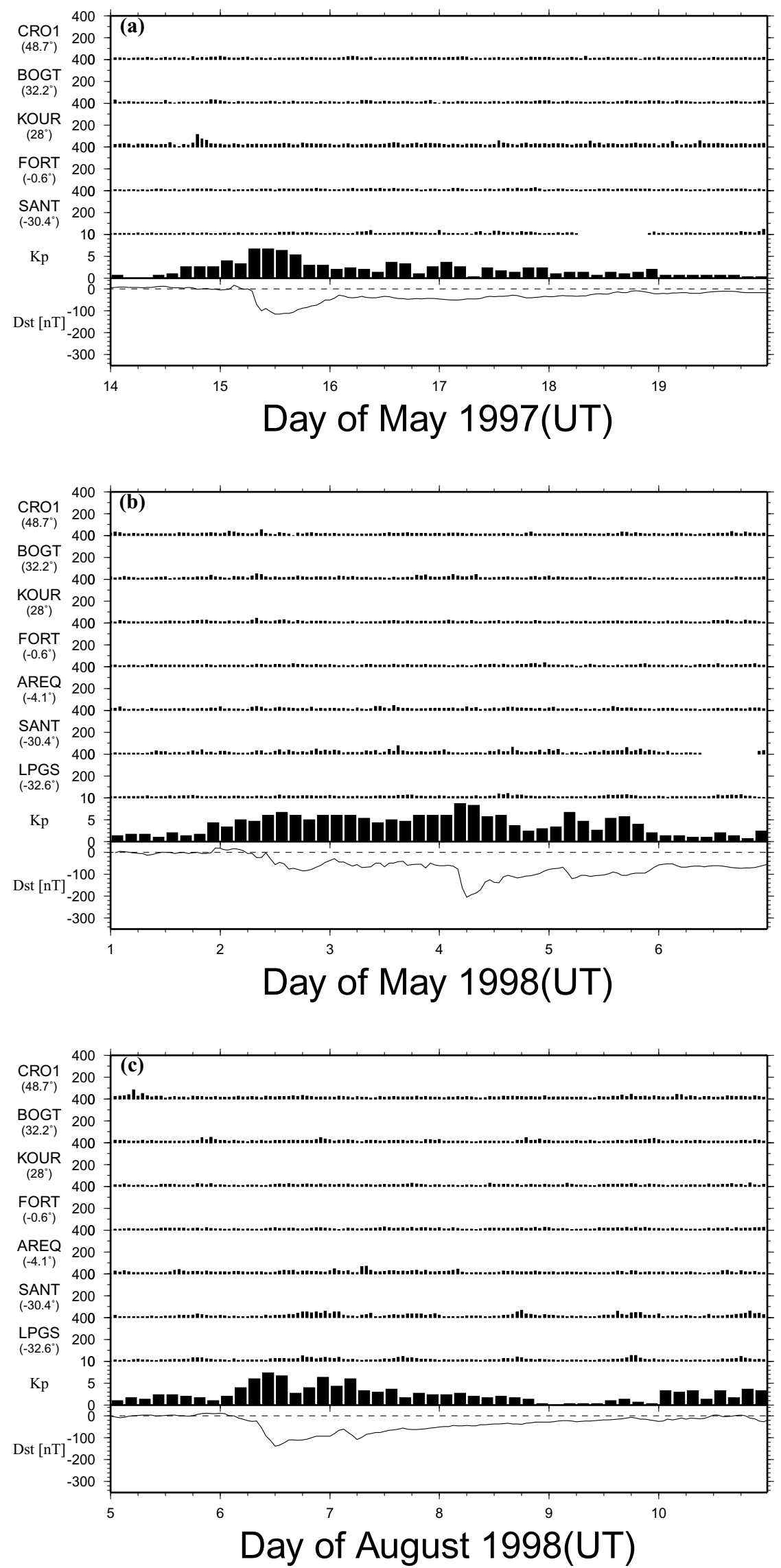

Fig. 3. The hourly $F p$ values and the associated $K p$ and $D s t$ indices of the magnetic storms from the GPS ground stations in Central-and South American sector occurring during 1997-2000. (a) Event A occurred on 15 May 1997, (b) Event B occurred on 1 May 1998, and (c) Event C occurred on 5 August 1998. 

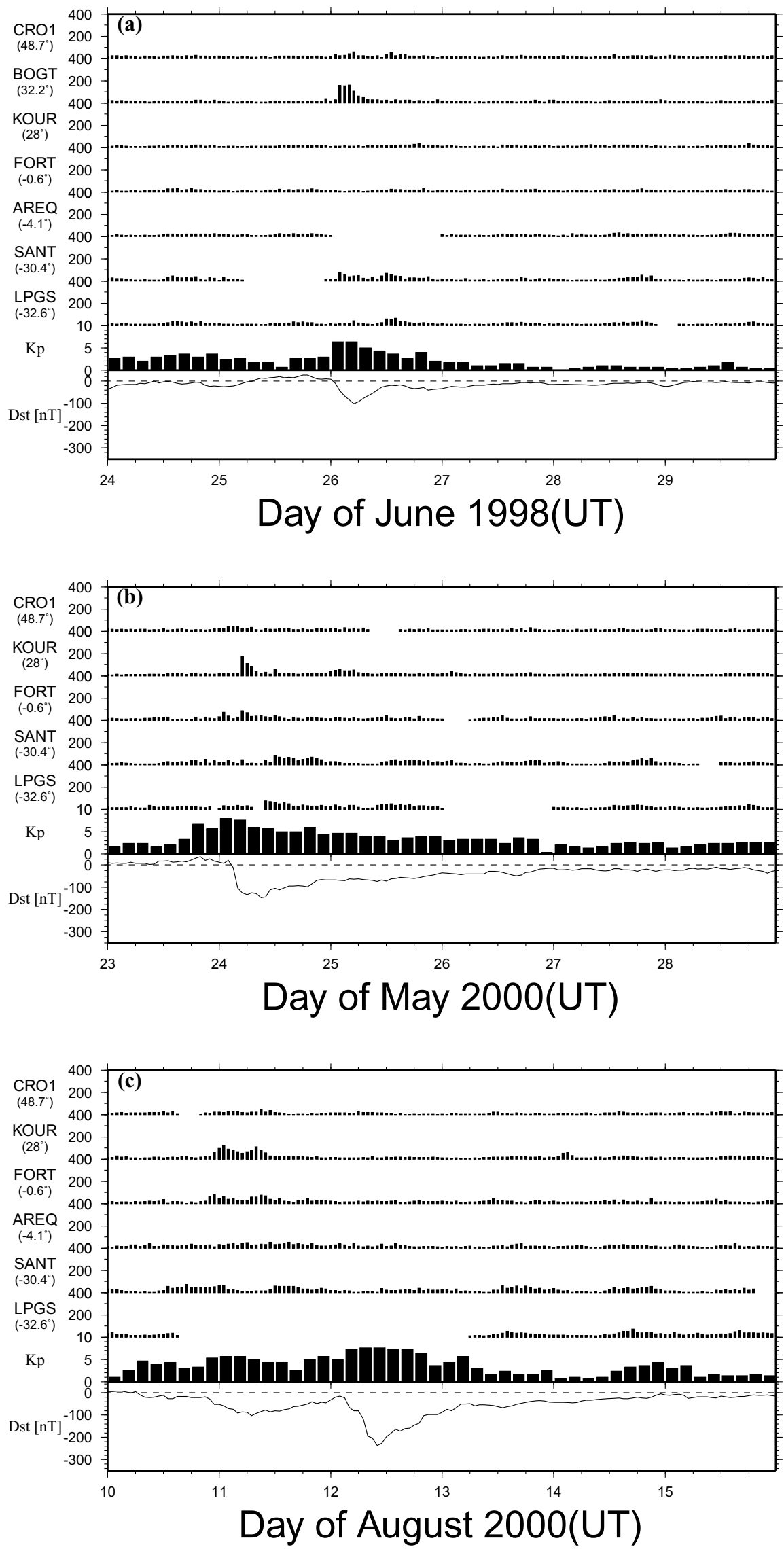

Fig. 4. (a) Event D occurred on 25 June 1998, (b) Event E occurred on 22 May 2000, and (c) Event F occurred on 10 August 2000. Layout is the same as that in Fig. 3. 

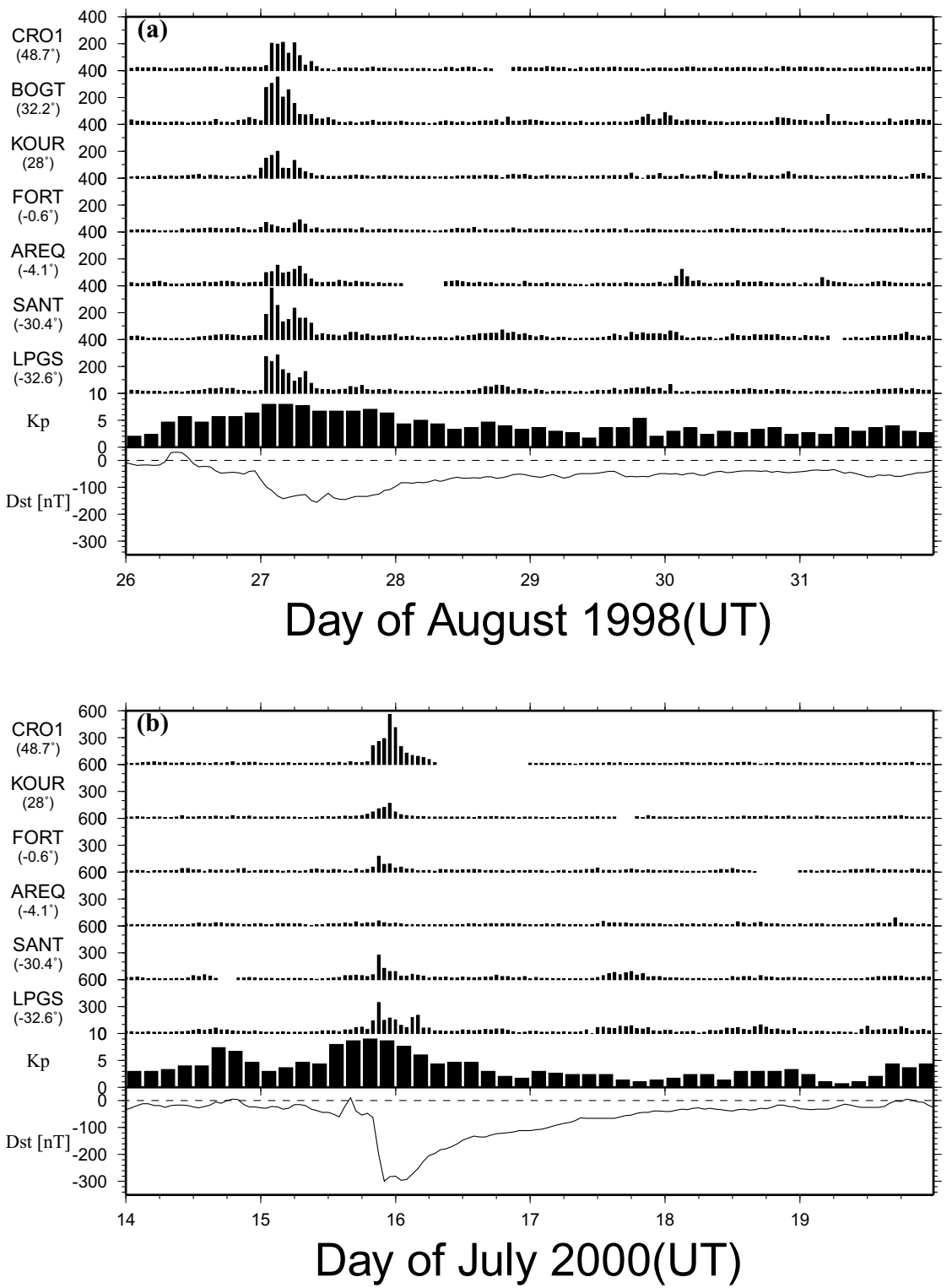

Fig. 5. (a) Event G occurred on 26 August 1998. (b) Event H occurred on 15 July 2000. Layout is the same as that in Fig. 3.

decrease after 0300 UT (around local midnight) on $12 \mathrm{Au}$ gust 2000 (Fig. 4(c)). It can be seen in the three events that the sharp decreases in the Dst index lead the increases of their associated phase fluctuations by about 2 hours. Meanwhile, the moderate phase fluctuations of the three events appear only at either BOGT or KOUR, which are located in the equatorial anomaly region of the northern (summer) hemisphere.

Two pronounced phase fluctuation Events $\mathrm{G}$ and $\mathrm{H}$ are observed in the evening hours. For Event G, significant phase fluctuations, $1 / 7 \mathrm{M}$ and $5 / 7 \mathrm{~S}$, started at $0100 \mathrm{UT}$ on 27 (about 2000-2300 LT on 26, post-sunset) August, while the Dst started to sharply decrease at 2300 UT on 26 August and reached its minimum at 1000 UT (about 0500-0800 LT, predawn) on 27 August 1998 (Fig. 5(a)). For Event H, significant phase fluctuations, $2 / 6 \mathrm{M}$ and $3 / 6 \mathrm{~S}$, began at $2000 \mathrm{UT}$ (about 1500-1800 LT, pre-sunset) 15 July, when the Dst began to drastically decrease at 1900 UT and reached its minimum at 2200 UT (about 1700-2000 LT, around post- sunset) 15 July 2000 (Fig. 5(b)). Once again, it can be seen in the two pronounced events that the starting times of the Dst decreases, Tb, lead the beginning time of the associated phase fluctuations, Tp, by about 1 or 2 hours. Meanwhile, it is clear in Figs. 5(a) and 5(b) that the magnitudes of phase fluctuations near the geomagnetic equator, FORT and AREQ, are relatively small but are gradually enhanced toward higher latitudes. The significant phase fluctuations appearing at SANT, CRO1, BOGT, LPGS, and KOUR (as far as $\pm 30^{\circ} \mathrm{N}$, geomagnetic) suggest that the irregularities extend to an extremely broad longitudes and latitudes.

Figures 6, 7, and 8 illustrate TEC contours (contour interval $=5$ TECU) derived from seven ground-based GPS stations during the eight storm periods. Here, the reference meridian is set at $300^{\circ} \mathrm{E}$ and interpolation is applied to locations where no observation is available. It is obvious that the TEC value at $\mathrm{Tb}$, when the Dst begins to sharply decrease, is relatively low (about 10-20 TECU) during Events A, B, and C (see Figs. 6(a), 6(b), and 6(c), respectively). 
Table 2. Summary of studied storm events.

\begin{tabular}{|c|c|c|c|c|c|c|}
\hline Event & $\begin{array}{l}\text { Magnetic storm Ts } \\
\text { dd/mm/yyyy hhmm }\end{array}$ & $\begin{array}{c}\mathrm{Tb} \\
\text { (UT) }\end{array}$ & $\begin{array}{l}\mathrm{Tm} \\
(\mathrm{UT})\end{array}$ & $\begin{array}{l}\text { Dst } \\
\text { (nT) }\end{array}$ & $\begin{array}{c}\text { Duration Tp } \\
\text { (UT) }\end{array}$ & $\begin{array}{c}F p \\
\text { (station) }\end{array}$ \\
\hline A & 15/05/19970300 UT & 0700 & 1300 & -115 & N/A & $0 / 5$ \\
\hline \multirow[t]{2}{*}{ B } & 01/05/1998 $2300 \mathrm{UT}$ & $1000^{*}$ & $1800^{*}$ & -85 & N/A & $0 / 7$ \\
\hline & & $0400^{* * *}$ & $0600^{* * *}$ & -205 & N/A & $0 / 7$ \\
\hline $\mathrm{C}$ & 05/08/1998 1800 UT & $0800^{*}$ & $1200^{*}$ & -138 & N/A & $0 / 7$ \\
\hline $\mathrm{D}$ & 25/06/1998 0800 UT & $0000^{*}$ & $0500^{*}$ & -101 & $0200^{*}$ (4 hrs) & 1/6M (BOGT) \\
\hline $\mathrm{E}$ & $22 / 05 / 20002000 \mathrm{UT}$ & $0300^{* *}$ & $0600^{* *}$ & -133 & $0500^{* *}(3 \mathrm{hrs})$ & $1 / 5 \mathrm{M}($ KOUR $)$ \\
\hline \multirow[t]{2}{*}{$\mathrm{F}$} & $10 / 08 / 20000600 \mathrm{UT}$ & 2100 & $0700^{*}$ & -103 & $2300(12 \mathrm{hrs})$ & 1/5M (KOUR) \\
\hline & & $0300^{* *}$ & $1000^{* *}$ & -237 & N/A & $0 / 5$ \\
\hline G & 26/08/1998 0800 UT & 2300 & $1000^{*}$ & -155 & $0100^{*}(6-9 \mathrm{hrs})$ & $5 / 7 \mathrm{~S}(\mathrm{BOGT})$ and $1 / 7 \mathrm{M}$ \\
\hline $\mathrm{H}$ & 15/07/2000 $1000 \mathrm{UT}$ & 1900 & 2200 & -300 & 2000 (3-10 hrs) & 3/6S (CRO1) and 2/6M \\
\hline
\end{tabular}

${ }^{*}$ day $+1,{ }^{* *}$ day $+2,{ }^{* * *}$ day +3

Accordingly, no clear phase fluctuations can be observed in Fig. 3. For Event D, when the TEC values are about 1020 and 10-15 TECU at Tb and Tp, respectively (Fig. 7(a)), the moderate phase fluctuations appear at BOGT around 0200 UT (Fig. 4(a)). Similarly, in Event E, the TEC values are around $25-50$ and $25-35$ TECU at Tb and Tp, respectively (Fig. 7(b)), and the short-duration phase fluctuations at KOUR start to increase moderately at 0500 UT (Fig. 4(b)). On the other hand, Event F has two Dst decreases (Fig. 7(c)). At the first Tb1, the TEC value is high (around 40-80 TECU) but only moderate phase fluctuations are observed at KOUR at 2300 UT and the TEC value at Tp is around 40-55 TECU (Fig. 4(c)). At the second Tb2, the TEC value is low (around 15-30 TECU) and no clear fluctuation can be detected (Fig. 4(c)).

For Event $\mathrm{G}$, the TEC values are both around 25-40 TECU at Tb and Tp (Fig. 8(a)). Note that the TEC value at $\mathrm{Tp}$ is more than double of that for the events $\mathrm{A}, \mathrm{B}, \mathrm{C}$, and $\mathrm{D}$ and significant phase fluctuations are observed at six ground-based GPS stations. Meanwhile the two equatorial anomaly crests move toward higher latitudes. For Event $\mathrm{H}$, the TEC value is around $70 \mathrm{TECU}$ at $\mathrm{Tb}$ and increases to 80 TECU at Tp when significant phase fluctuations start (see Fig. 8(b)). The two equatorial anomaly crests again move toward higher latitudes with great TEC values (around 85100 TECU). The TEC value of the southern crest of the equatorial anomaly is larger than that of the north, which is consistent with strong phase fluctuations pattern displayed in Fig. 5(b). Meanwhile, it is found in Figs. 5(a) and 5(b) that the magnitudes of phase fluctuations near the geomagnetic equator are relatively small but gradually enhance toward higher latitudes. The similar trend can be found in Figs. 8(a) and 8(b) that the two crests expand toward higher latitudes and their associated TEC values increase.

\section{Discussion and Conclusion}

In this study, we investigate the GPS phase fluctuations observed during the months of low irregularity activity, and therefore that no regular development of irregularities is expected to occur in the evening hours (Aarons, 1993;
Wanninger, 1993). This allows us to discriminate the seasonal and storm factors, thus having a better chance to understand the origin of the GPS phase fluctuations. Aarons (1993), Maruyama and Matuura (1984), Maruyama (1988), and Wanninger (1993) found that the occurrence frequencies of ionospheric irregularities in South America are significantly lower during the winter season (i.e., May-August) than during summer. Figure 2 also illustrates that during May-August the Fps generally yield smaller values, which is in excellent agreement with previous observations.

Though KOUR and BOGT located in similar geomagnetic latitudes (Table 1), the amplitude of mean $F p$ of the two stations are significantly different (see Fig. 2). The similar situation is also found between FORT and AREQ. Mendillo et al. (2000) reported the similar tendency that the phase fluctuations of the stations on the east-side of the south America tend to be larger than that of the westside stations. However, the asymmetry between the eastside and west-side of the South America is not seen in the storm periods (Fig. 5(a)). This is probably due to the background conditions of the ionosphere that are not the same between the storm periods and quiet days. Meanwhile from Fig. 2, though BOGT and SANT have similar geomagnetic dips, phase fluctuation activity at BOGT is larger than that at SANT. The stations in the northern hemisphere tend to have higher activity than those in the southern hemisphere. The asymmetry between the magnetic northern and southern hemispheres is also observed in the storm periods (Figs. 5(a) and 5(b)).

Scientists have examined the sequential relationship between magnetic storms and phase fluctuations. It has been known that during the months of high occurrence frequency of the ionospheric irregularities, phase fluctuations are usually observed between 1900 LT (post-sunset) and 0100 LT (midnight). For geomagnetic storms, Aarons (1991) further found that if the Dst value reaches a minimum between 1800 and 2200 LT (post-sunset), the duration of the observed fluctuations would last for several hours. In contrast, if the Dst minimum appears during 0000-0600 LT (post-midnight to pre-dawn), then two consecutive periods 

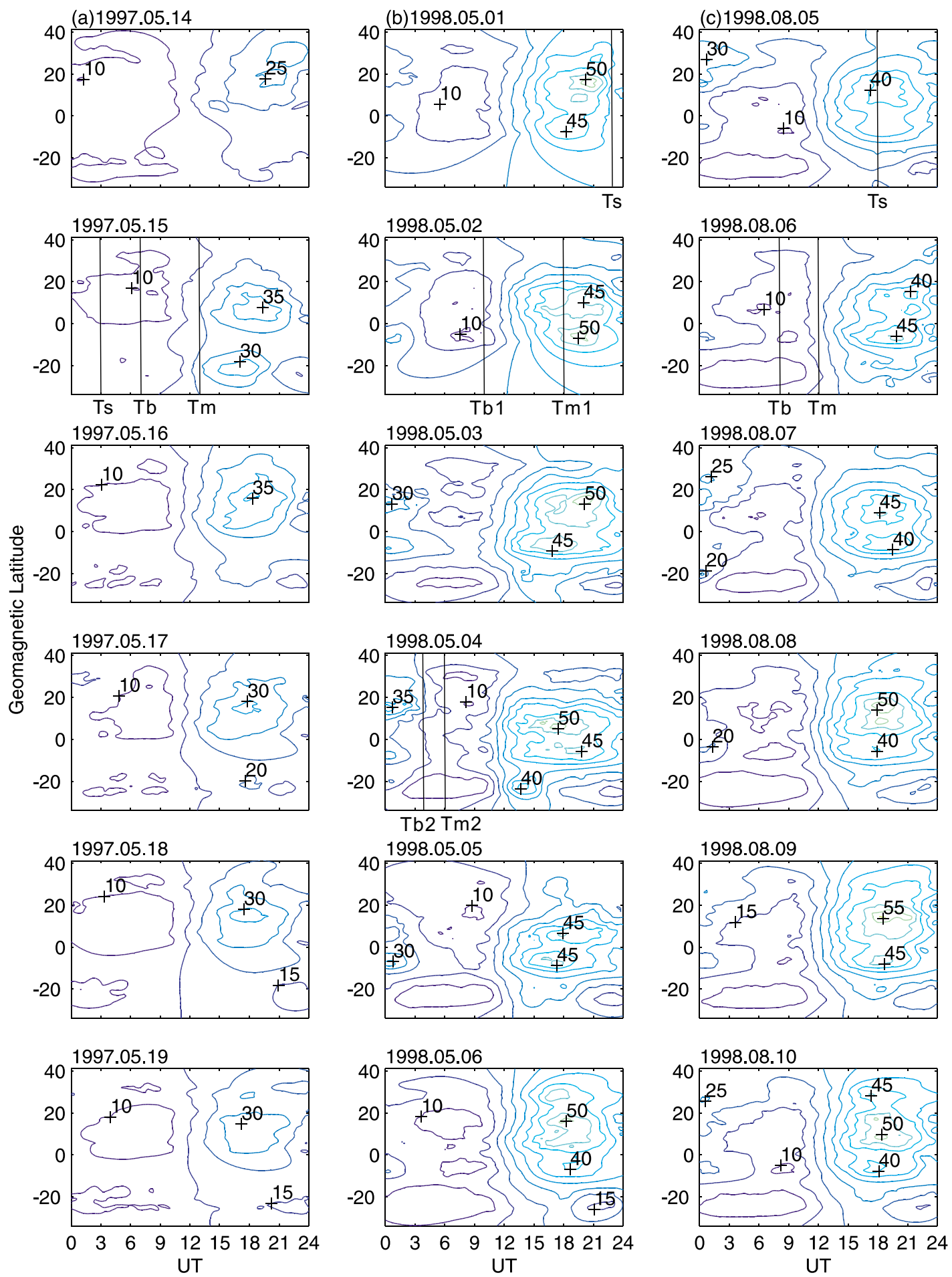

Fig. 6. TEC contours for magnetic storms in this study. Contour interval is 5 TECU. (a) Event A occurred on 15 May 1997. (b) Event B occurred on 1 May 1998. (c) Event C occurred on 5 August 1998.

of phase fluctuations would be observed. The former corresponds to the regular one appearing immediately after local sunset, while the latter takes place synchronously with the Dst minimum. On the other hand, there would be no phase fluctuations observed if the Dst minimum occurs in the daytime (i.e., between 1000 and 1600 LT).

Figures 5(a) and 5(b) show that the phase fluctuations of the two pronounced events appeared at regular times as in the month of high irregularity activity, rather than during the time when the Dst reached its minimum. Meanwhile, the $\mathrm{Tb}$ and $\mathrm{Tp}$ listed in Table 2 suggest that during the low irregularity activity months, the ionospheric phase fluctuations are easily triggered by the magnetic storm when the Dst begins to sharply decrease between 2000 UT to 2300 UT (1500 LT to 2100 LT, pre- and post-sunsets). If the Dst begins to decrease sharply around local midnight, there is some chance to observe phase fluctuation only at station BOGT or KOUR (Events D and E). If the Dst begins to 

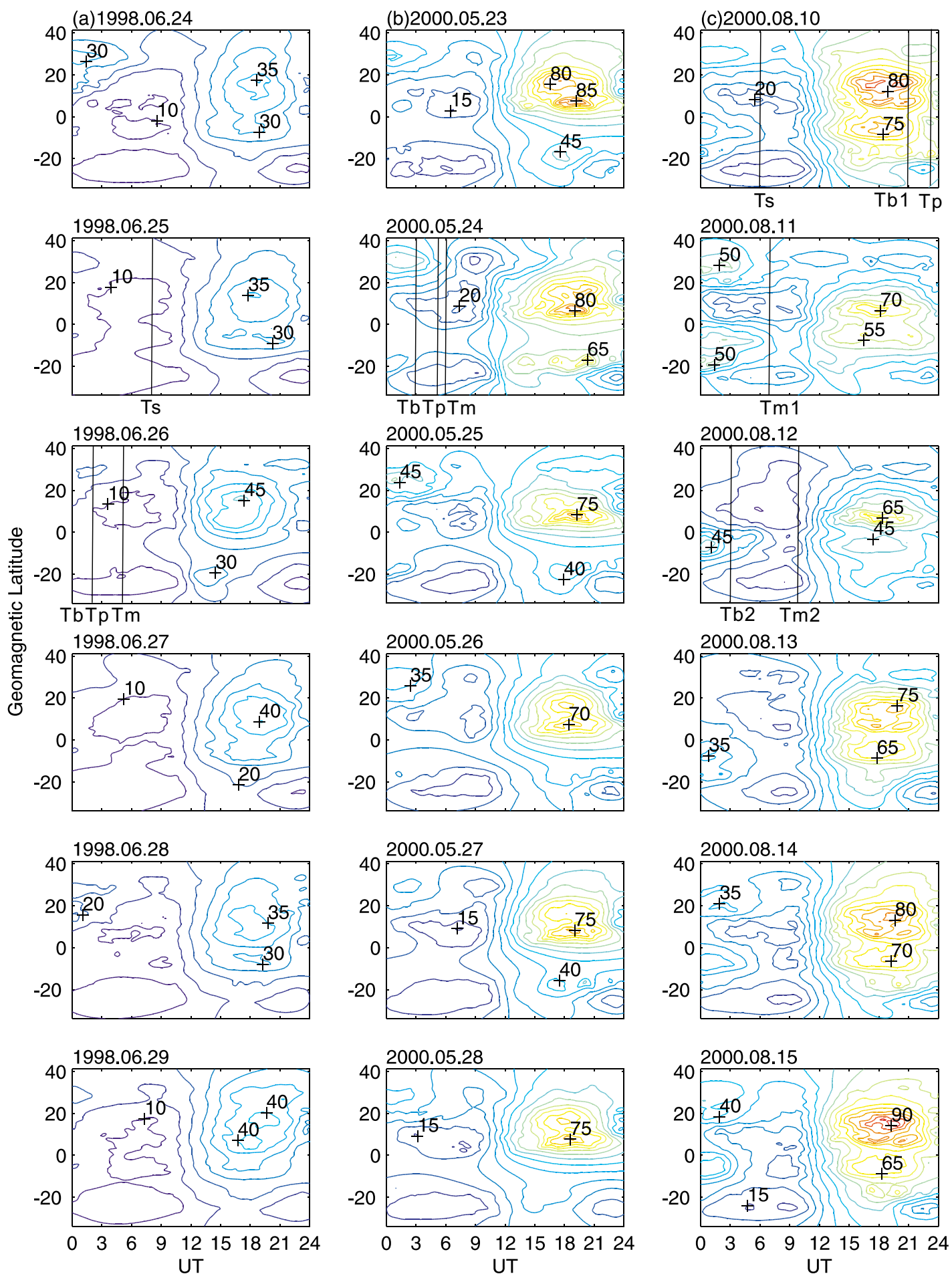

Fig. 7. (a) Event D occurred on 25 June 1998. (b) Event E occurred on 22 May 2000. (c) Event F occurred on 10 August 2000. Layout is the same as that in Fig. 6.

decrease after local midnight, then no phase fluctuation is observed (Events A, B, and C). For Event F, even though the Dst first starts to decrease at 2100 UT (1600 to 1900 LT) on 10 August 2000, which lies within a favorable time period, the strength was relatively small $(-103 \mathrm{nT})$, thus the phase fluctuation appears only at KOUR. In contrast, the second Dst with the minimum of about $-237 \mathrm{nT}$, starts to decrease around post-midnight on 12 August, which falls on the unfavorable local time, and therefore no significant phase fluctuation is observed.

The descent in Event E (Tb: 0300 UT, 25-50 TECU, Fig. 7(b)), and the second descents in Event B (Tb2: 0400 UT, 10-20 TECU, Fig. 6(b)), and in Event F (Tb2: 0300 UT, $\sim 15-30$ TECU, Fig. 7(c)) all occurred during the similar local time, but the phase fluctuations appeared only in Event E whose Dst variation was the smallest among these three events. The descending speed of the second Dst in Event $\mathrm{F}$ is slightly smaller than that in Event $\mathrm{E}$ and the 

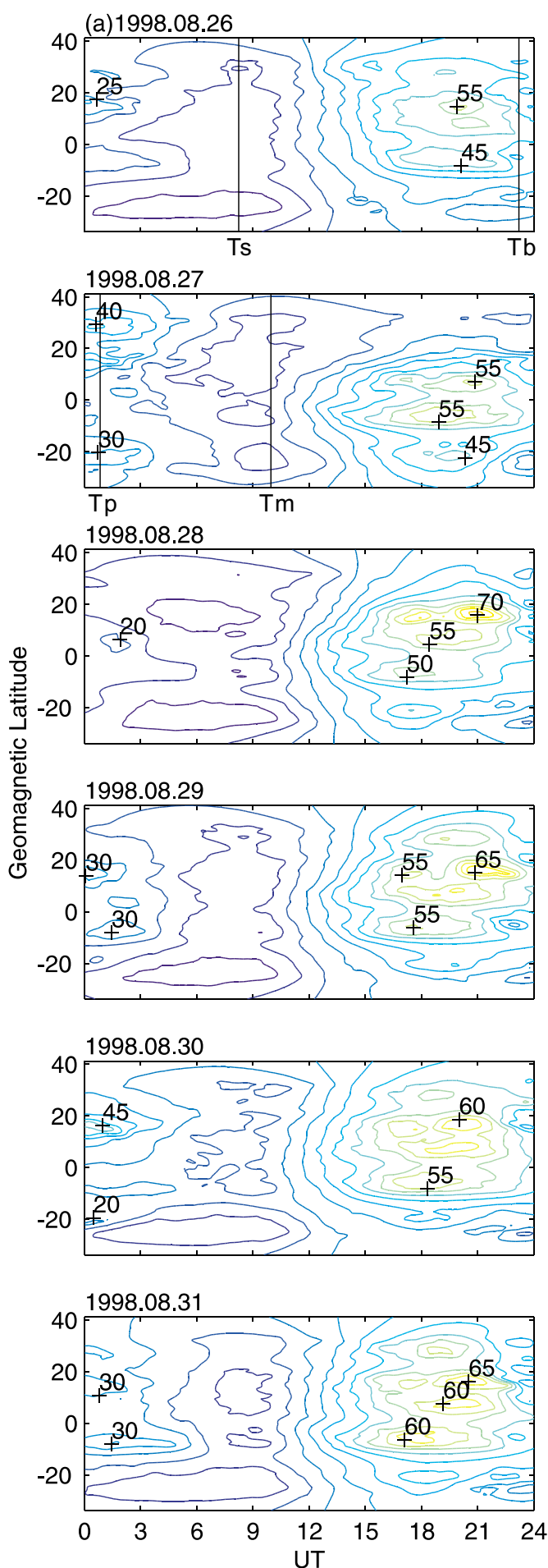
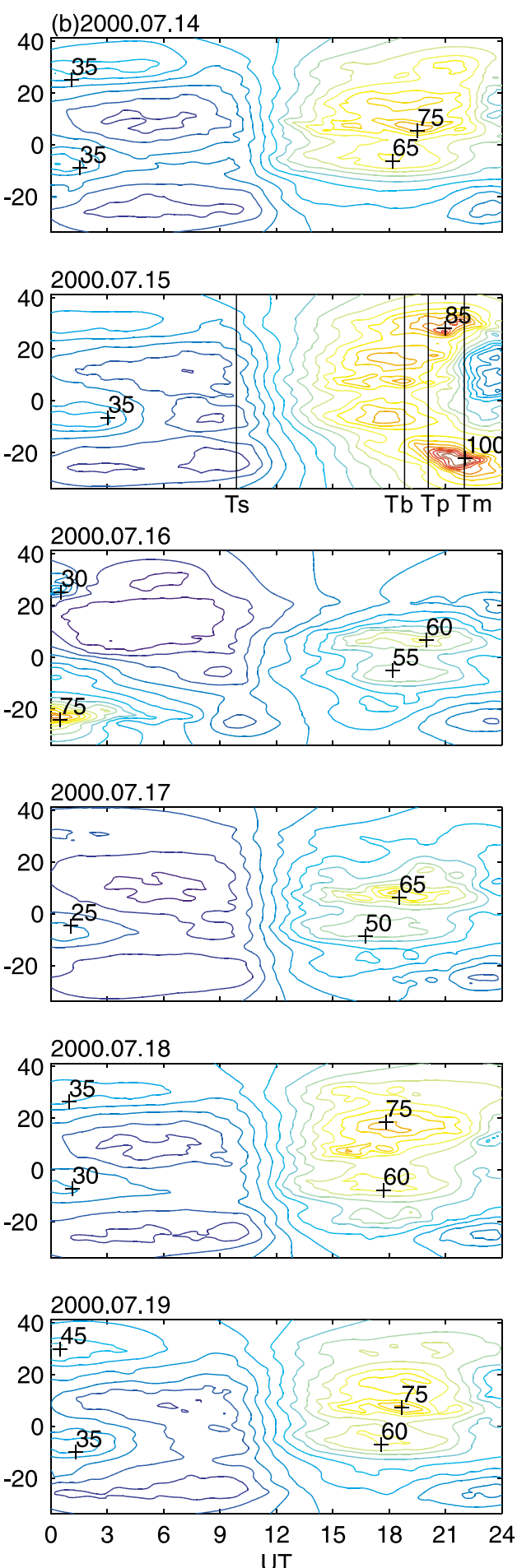

Fig. 8. (a) Event G occurred on 26 August 1998. (b) Event H occurred on 15 July 2000. Layout is the same as that in Fig. 6.

second one in Event B. Furthermore, among the three examples when Dst begins to sharply decrease (Tb), the TEC value of Event $\mathrm{E}$ yields the largest value. This indicates that the generation of phase fluctuations is more correlated with the large crest TEC values rather than the storm intensity measured by Dst index.

During quiet time periods, phase fluctuations at CRO1 are low in amplitude, as shown in Fig. 2. This would be caused by the fact that CRO1 is located at higher latitude than the equatorial anomaly crests and out of the influence of the fountain effect. By contrast, during the storm periods, CRO1 also detected the phase fluctuations (Figs. 5(a) and 5(b)). From Figs. 8(a) and 8(b), both Events $G$ and $H$ have the largest TEC values at the starting time of phase fluctuation, $\mathrm{Tp}$, and also the two peaks of the equatorial anomaly expand into higher latitudes. This implies that the intense eastward electric field raises the plasma to the higher altitude than in the quiet period at the equator. This effect may push the equatorial anomaly to higher latitudes than the location in the quiet time. 
It has been suggested by scientists (Jones and Rishbeth, 1971; Blanc and Richmond, 1980; Gonzales et al., 1983; Kelley et al., 1979; Sastri et al., 1992) that a storm can directly or indirectly produce an intense eastward electric field which uplifts ionospheric plasma from the equatorial F-layer to higher altitudes and then diffuses downward to and pile up in the low-latitude off-equatorial regions, which results in an instability of the ionosphere and triggers irregularities (or phase fluctuations). The strong phase fluctuations that often appear at KOUR and BOGT, which are just under the equatorial anomaly region $\left( \pm 15^{\circ} \mathrm{N}\right.$, geomagnetic) and are significantly affected by the fountain effect of E_B drift, indicate that the eastward electric field could play an important rule. The latitude dependence of the phase fluctuation amplitude during the over all and the storm periods can be found in Figs. 2 and 5, respectively. The clear latitude dependence could be explained by the enhancement of the equatorial anomaly during the storm periods. It is the intense eastward electric field imposed by the geomagnetic storm transports the plasma at the dip equator to the off-equatorial region (Jakowski et al., 1990; Batista et al., 1991).

Batista et al. (1986), Fejer et al. (1989) and Batista et al. (1991) examined an increase of the prereversal enhancement of the upward drifts with magnetic activities, and found the correlation between magnetic storms and TEC increase in low latitudes. Figs. 8(a) and 8(b) illustrate that the TEC values at the time Tp of Event $\mathrm{G}$ and $\mathrm{H}(\sim 25-40$ and $\sim 55-80$ TECU, respectively) are much greater than most events (except for only one case, Event G vs. Event F) and also the two equatorial anomaly crests expand into higher latitudes, suggesting that the intense eastward electric field causes the vertical drift and uplifts of ionospheric plasma to higher altitudes. Due to the smaller recombination losses and the efficient diffusion at higher altitudes, a great amount of ionization is transported from the equatorial and low latitude regions to higher latitudes, thus resulting in the expansion of the TEC anomaly (i.e., the equatorial ionization anomaly, EIA). It might be that the expansion of EIA and the associated larger TEC value provide favorable conditions for the development of plasma instabilities (or GPS phase fluctuations) (Kelley, 1989).

In conclusion, we show that there are two predominant factors in generating irregularities in a low occurrence season: (1) the local time of the magnetic Dst index that begins to sharply decrease, and (2) the existence of relatively intense TEC crests. Specifically, the Dst index must begin to sharply decrease during pre- and post-sunset periods, and also the storm must induce an intense eastward electric field in the equatorial regions to cause an unstable environment to let gravity wave or other seeding.

Acknowledgments. This research has been partially supported by National Science Council of ROC Grants NSC89-2111-M-008-051 and NSC89-2111-M-008-055 to National Central University. The authors would like to express their appreciation to the International GPS Service (IGS) and WDC-C2 Kyoto Dst index service for the data provided. Figures 1, 3, 4, and 5 were generated by the GMT software written by Paul Wessel at University of Hawaii and Walter H. F. Smith at NOAA. The authors thank Dr. Chin-Chun Wu at NASA for the graphic and software assistance. Constructive comments from two anonymous reviewers are gratefully acknowledged.

\section{References}

Aarons, J., The role of the ring current in the generation or inhibition of equatorial $\mathrm{F}$ layer irregularities during magnetic storms, Radio Sci., 26, 1131-1149, 1991.

Aarons, J., The longitudinal morphology of equatorial F-layer irregularities relevant to their occurrence, Space Sci. Rev., 63, 209-243, 1993.

Aarons, J., M. Mendillo, and R. Yantosca, GPS phase fluctuations in the equatorial region during the MISETA 1994 campaign, J. Geophys. Res., 101, 26,851-26,862, 1996

Aarons, J., M. Mendillo, and R. Yantosca, GPS phase fluctuations in the equatorial region during sunspot minimum, Radio Sci., 32, 1535-1550, 1997.

Basu, Su., E. MacKenzie, and S. Basu, Ionospheric constraints on $\mathrm{VHF} / \mathrm{UHF}$ communication links during solar maximum and minimum periods, Radio Sci., 23, 363-378, 1988.

Batista, I. S., M. A. Abdu, and J. A. Bittencourt, Equatorial F region vertical plasma drifts: Seasonal and longitudinal asymmetries in the American sector, J. Geophys. Res., 91, 12,055-12,064, 1986.

Batista, I. S., E. R. D. Paula, M. A. Abdu, and N. B. Trivedi, Ionospheric effects of the March 13, 1989, Magnetic storm at low and equatorial latitudes, J. Geophys. Res., 96, 13,943-13,952, 1991.

Beach, T. L. and P. M. Kintner, Simultaneous global positioning system observations of equatorial scintillations and total electron content fluctuations, J. Geophys. Res., 104, 22,553-22,565, 1999.

Blanc, M. and A. D. Richmond, The ionospheric disturbance dynamo, $J$ Geophys. Res., 85, 1669-1686, 1980.

Dabas, R. S., D. R. Lakshmi, and B. M. Reddy, Effect of geomagnetic disturbances on the VHF nighttime scintillation activity at equatorial and low latitudes, Radio Sci., 24, 563-573, 1988.

Fejer, B. G., E. R. D. Paula, I. S. Batista, E. Bonelli, and R. F. Woodman, Equatorial $\mathrm{F}$ region vertical plasma drifts during solar maxima, J. Geophys. Res., 94, 12,049-12,054, 1989.

Gonzales, C. A., M. C. Kelley, R. A. Behnke, J. F. Vickrey, R. Wand, and J. Holt, On the latitudinal variations of the ionospheric electric field during magnetospheric disturbances, J. Geophys. Res., 88, 9135-9144, 1983.

Ho, C. M., A. J. Mannucci, U. J. Lindqwister, X. Pi, and B. T. Tsurutani, Global ionosphere perturbations monitored by the worldwide GPS network, Geophys. Res. Lett., 23, 3219-3222, 1996.

Jakowski, N., E. Putz, and P. Spalla, Ionospheric storm characteristics deduced from satellite radio beacon observations at three European stations, Ann. Geophys., 8, 343-352, 1990.

Jakowski, N., S. Schluter, and E. Sardon, Total electron content of the ionosphere during the geomagnetic storm on 10 January 1997, J. Atmos. Solar-Terr. Phys., 61, 299-307, 1999.

Jones, K. L. and H. Rishbeth, The origin of storm increases of mid-latitude F-layer electron concentration, J. Atmos. Terr. Phys., 33, 391-401, 1971.

Kelley, M. C., The Earth's Ionosphere, 487 pp., Academic Press, San Diego, 1989.

Kelley, M. C., B. G. Fejer, and C. A. Gonzales, An explanation for anomalous ionospheric electric fields associated with a northward turning of the interplanetary magnetic field, Geophys. Res. Lett., 6, 301-304, 1979.

Leick, A., GPS Satellite Surveying, 560 pp., John Wiley \& Sons, New York, 1995

Liu, J. Y., H. F. Tsai, and T. K. Jung, Total electron content obtained by using the global positioning system, Terr. Atmos. Oceanic Sci., 7, 111$121,1996$.

Liu, J. Y., H. F. Tsai, C. C. Wu, C. L. Tseng, L. C. Tsai, W. H. Tsai, K. Liou, and J. K. Chao, The effect of geomagnetic storm on ionospheric total electron content at equatorial anomaly region, Adv. Space Res., 24, 1491-1494, 1999

Maruyama, T., A diagnostic model for equatorial spread F: 1. Model description and application to electric field and neutral wind effects, $J$. Geophys. Res., 93, 14,611-14,622, 1988.

Maruyama, T. and N. Matuura, Longitudinal variability of annual changes in activity of equatorial spread $\mathrm{F}$ and plasma bubbles, J. Geophys. Res., 89, 10,903-10,912, 1984.

Mendillo, M., B. Lin, and J. Aarons, The application of GPS observations to equatorial aeronomy, Radio Sci., 35, 885-904, 2000.

Mullen, J. P., Sensitivity of equatorial scintillation to magnetic activity, $J$. Atmos. Terr. Phys., 35, 1187-1194, 1973.

Pi, X., A. J. Mannucci, U. J. Lindqwister, and C. M. Ho, Monitoring of global ionospheric irregularities using the worldwide GPS network, 
Geophys. Res. Lett., 24, 2283-2286, 1997.

Sardón, E., A. Rius, and N. Zarraoa, Estimation of the transmitter and receiver differential biases and the ionospheric total electron content from Global Positioning System observations, Radio Sci., 29, 577-586, 1994.

Sastri, J. H., K. B. Ramesh, and D. Karunakaran, On the nature of substormrelated transient electric field disturbances in the equatorial ionosphere, Planet. Space Sci., 40, 95-103, 1992.

Tsai, H. F. and J. Y. Liu, Ionospheric total electron content response to solar eclipses, J. Geophys. Res., 104, 12,657-12,668, 1999.

Wanninger, L., Effects of the equatorial ionosphere on GPS, GPS World (July), 48-54, 1993.

Zarraoa, N. and E. Sardón, Test of GPS for permanent ionospheric TEC monitoring at high latitudes, Ann. Geophys., 14, 11-19, 1996.

S. J. Shan (e-mail: shan@aeolus.earth.sinica.edu.tw), J. Y. Liu, F. S. Kuo, C. C. Liu, and H. F. Tsai 